\title{
The genetic architecture of sexually selected traits in two natural populations of Drosophila montana
}

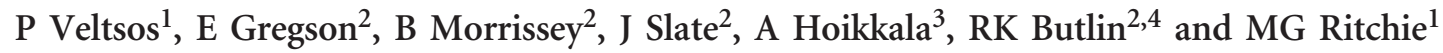 \\ We investigated the genetic architecture of courtship song and cuticular hydrocarbon traits in two phygenetically distinct \\ populations of Drosophila montana. To study natural variation in these two important traits, we analysed within-population \\ crosses among individuals sampled from the wild. Hence, the genetic variation analysed should represent that available for \\ natural and sexual selection to act upon. In contrast to previous between-population crosses in this species, no major \\ quantitative trait loci (QTLs) were detected, perhaps because the between-population QTLs were due to fixed differences \\ between the populations. Partitioning the trait variation to chromosomes suggested a broadly polygenic genetic architecture of \\ within-population variation, although some chromosomes explained more variation in one population compared with the other. \\ Studies of natural variation provide an important contrast to crosses between species or divergent lines, but our analysis \\ highlights recent concerns that segregating variation within populations for important quantitative ecological traits may largely \\ consist of small effect alleles, difficult to detect with studies of moderate power.
}

Heredity (2015) 115, 565-572; doi:10.1038/hdy.2015.63; published online 22 July 2015

\section{INTRODUCTION}

Quantitative trait locus (QTL) studies are typically performed by identifying lines or species that differ in a trait of interest, crossing them and correlating the phenotypic variation in the resulting offspring with genetic variation, to pinpoint genomic regions associated with the trait of interest. If the trait of interest is thought to be important to speciation, the initial cross is often made between inbred lines from different species or differentially adapted populations. However, using highly differentiated populations cannot indicate whether the same differences were involved in the initial isolation of the populations, because current differences may have arisen since the populations became isolated. In fact, it seems that the types of mutations providing an initial response to selection within species may differ from those eventually fixed between species (Stern and Orgogozo, 2008; 2009). QTL studies should, therefore, be performed within and between populations representing multiple divergence times.

More generally, the range of trait variation captured by the initial cross will influence both the ability to detect QTL and possibly the nature and the effect size of QTLs detected. Choosing very different individuals for the initial cross may identify large effect QTLs, which may not be segregating in wild populations. The effectiveness of the candidate gene approach is a related issue. Candidate genes are identified by their large effects and, if their function is evolutionarily conserved, they should be associated with variation in the same function in different species. Such loci have been found for a variety of traits, including behaviour patterns (Fitzpatrick et al., 2005; Martin and Orgogozo, 2013). In other cases, a polygenic genetic architecture is reported, for example, in recent QTL studies of ecologically important traits (Davies et al., 2011; Rockman, 2012; Travisano and Shaw, 2013). This has led some to question the value of looking for large effect loci for understanding ecological adaptation within species. Even where large effect loci are segregating, they may be at a very low frequency leading to only a small contribution to the population-level heritability of such traits (Scoville et al., 2011). It is therefore important to compare the genetic architecture of traits in different types of crosses, such as within vs between populations or lab- vs fieldreared, and assess how often similar or different genetic effects are found.

Studies of the quantitative genetics of ecologically important traits in the field are proceeding well for some vertebrates with extensive pedigree data (Kruuk and Hill, 2008; Robinson et al., 2013) and some important segregating loci have been identified (Johnston et al., 2011), but there are very few studies for quantitative traits in species such as Drosophila that are the source of much of the information on species differences. The circum-arctic species Drosophila montana has proved to be a good species to study adaptive traits, including cold tolerance, developmental time, juvenile body weight and reproductive diapause (Vesala and Hoikkala, 2011; Salminen et al., 2012; Parker et al., 2015) in the wild. Phylogeographic analysis of the species based on mitochondrial DNA and microsatellites suggests two clades, a North-American and European, that were isolated about $0.5 \mathrm{Myr}$ ago (Mirol et al., 2007). North-American D. montana populations have been classified as Standard, Giant and Alaskan-Canadian based on their geographical origin, size and chromosome structure (Throckmorton, 1982), and Eurasian populations contain several unique inversions (Morales-Hojas et al., 2007). There is evidence for pre-zygotic isolation (assortative mating), post-mating pre-zygotic

${ }^{1}$ Centre for Biological Diversity, University of St Andrews, St Andrews, UK; ${ }^{2}$ Animal \& Plant Sciences, University of Sheffield, Alfred Denny Building, Sheffield, UK; ${ }^{3}$ Department of Biological and Environmental Science, University of Jyväskylä, Jyväskylä, Finland and ${ }^{4}$ Sven Lovén Centre-Tjärnö, University of Gothenburg, Strömstad, Sweden Correspondence: Dr P Veltsos, Centre for Biological Diversity, University of St Andrews, Dyers Brae House, St Andrews, KY16 9TH, UK. E-mail: parisveltsos@gmail.com

Received 12 December 2014; revised 26 April 2015; accepted 5 June 2015; published online 22 July 2015 
isolation (successful sperm transfer but failure to fertilise eggs) and post-mating post-zygotic isolation (progeny production) between some populations (Jennings et al., 2011; Jennings et al., 2014b). The main reasons to classify these populations as belonging to the same species are that the strength of these different barriers to gene flow depends on the population pairs being crossed and that pre-mating isolation is only evident when females can choose between a male from their own population and another population. Population differences therefore fall into the rarely studied early part of the speciation continuum (Safran et al., 2013).

There have been extensive studies of within- and between-species phenotypic variation in the sexual behaviour of the virilis group to which $D$. montana belongs. Male courtship song is essential for mating in D. montana (Liimatainen et al., 1992), has a species-specific component, inter-pulse interval (IPI) (Liimatainen and Hoikkala, 1998; Saarikettu et al., 2005a), and crosses between closely related species have indicated X-linkage for genes influencing some song traits (Hoikkala et al., 2000; Päällysaho et al., 2003). Courtship song is under sexual selection and seems to be an honest indicator of male quality: one song component, intra-pulse frequency (FRE), is conditiondependent, correlated with offspring survival and under strong and contrasting viability and sexual selection in the field (Aspi and Hoikkala, 1995; Hoikkala et al., 1998). Various song components influence mating latency and courtship duration (Veltsos et al., 2012), and populations differ in both song and preference (Klappert et al., 2007; Ritchie et al., 2007). Recent studies of cuticular hydrocarbons (CHCs) in D. montana show that they influence mating decisions independently of song and also differ between populations (Veltsos et al., 2012; Jennings et al., 2014a).

We previously analysed the influence of song and $\mathrm{CHC}$ variation in male mating success in $D$. montana through no-choice experiments where females were presented with a male from the same population (Oulanka, Finland or Vancouver, Canada) and the mating occurrence, mating latency and female rejection song production were correlated with various components of male courtship song and CHCs (Veltsos et al., 2012). In this paper, we complete quantitative genetic and QTL analyses of the same traits by investigating and comparing their genetic architecture. The same two independent populations, representing distinct phylogeographic clades (Oulanka and Vancouver) were used. The phenotypic data of both populations were jointly analysed with principal component analysis (PCA), so that the populations can be compared. We compare the within-population QTL analysis of song variation with previous work that identified QTL influencing courtship song by using between-population outbred crosses of D. montana (from Oulanka, Finland and Colorado, N. America). The detected QTL were on two chromosomes (X and 2) and one candidate gene was potentially associated with each QTL (per and fru, respectively) (Schafer et al., 2010; Lagisz et al., 2012), although it has not been confirmed whether these or other genes under the peaks are responsible for the phenotypic effects.

Here, we investigate whether within-population variation in the same traits involves the same genomic regions as the betweenpopulation analysis. We established two independent pedigrees from wild collected females, developed single nucleotide polymorphism (SNP) markers by transcriptome sequencing and scored individuals from the pedigrees for SNPs and song and $\mathrm{CHC}$ traits. Most traits exhibited moderate heritability. Our QTL and chromosome partitioning results reveal no large effects in the regions identified by the between-population crosses, and suggest differences in genetic architecture between the populations in terms of the proportion of additive genetic variation explained by each chromosome. Overall, they highlight the difficulty of gaining adequate power to detect loci of small effect in studies on the genetic architecture of wild populations.

\section{MATERIALS AND METHODS}

\section{Sampling and phenotyping}

Field sampling, cross design and phenotyping have been described previously (Veltsos et al., 2012). Briefly, we constructed and collected phenotypic and genotypic information on two or three generation pedigrees of about 500 individuals each from the offspring of wild-caught females from Vancouver, Canada and Oulanka, Finland. There were 30 isofemale lines from Vancouver and 42 from Oulanka. Each line may have had more than one father, as the frequency of multiple mating in the wild has been estimated as $1.19 \pm 0.31$ (Aspi, 1992). Song recording took place while setting up the crosses of the next generation. We measured four song parameters: intra-pulse frequency (FRE), inter-pulse interval (IPI), pulse number and cycle number per pulse (CN). We analysed these traits and the principal components (PCs) of song variation (using data from both populations so that the PCs are directly comparable), including recording temperature as a covariate because it has a strong effect on song (Ritchie et al., 2001).

CHCs were extracted from whole individuals of both sexes, after the females had laid sufficient eggs to provide individuals for the next pedigree generation (>20 eggs). PCs of CHC data were obtained from both sexes simultaneously because they are only moderately sexually dimorphic in $D$. montana, with no sex-specific compounds (Veltsos et al., 2012; Jennings et al., 2014a). The PCA was on relative proportions of log-transformed CHCs (Rundle et al., 2009) and used 18 compounds in total. PCA was performed on data from both populations simultaneously, to make them directly comparable in QTL analysis. A full description of the PCA, characterisation of population differences in CHCs as well as their association with measures of fitness in the lab are presented in Veltsos et al. (2012).

\section{Transcriptome sequencing}

RNA was extracted from 120 heads of 2-3-week-old virgin adults of both sexes from offspring of the last generation of each pedigree, using a Qiagen RNA extraction kit (Qiagen, Hilden, Germany), following the manufacturer's instructions and including on-column DNA digestion. RNA samples were processed by Genepool in Edinburgh (Vancouver flies) and the Centre for Genomics Research in Liverpool (Oulanka flies). Complementary DNA libraries were constructed without normalisation and were sequenced on half a 454 pyrosequencing plate each (Roche, FLX, Bradford, CT, USA).

The sequencing reads were filtered to remove low quality sequence (threshold of 20) and trimmed to remove adaptor, primer sequence and poly-A tails using SeqMan NGen version 1.2 (DNASTAR, Madison, WI, USA); 348 267/417 467 and $804456 / 1211678$ assembled/total reads were available for Vancouver and Oulanka, respectively. Significantly more reads were available for Oulanka because two sequencing runs were combined (the first run yielded only a few reads). An initial assembly was made by combining all reads from both populations. The resulting master contigs were used to guide two assemblies, one for each transcriptome. This ensured easy comparison of SNP locations from each population. All assemblies were performed with SeqMan NGen version 1.2 (DNASTAR).

\section{SNP marker development}

We aimed to obtain SNP markers that segregated in both populations. The best 384 SNPs to score were identified through custom scripts, for details see Data Archiving. Briefly, SNPs were called in each population using SeqMan NGen version 1.2 (DNASTAR) and categorised as being unique to a population, on the same contig, or identical between populations. Criteria for shortlisting SNPs were good coverage (at least 8 reads in total and 3 reads or $20 \%$ for the minor allele), long distance from other SNPs (at least $25 \mathrm{bp}$ ), scoring primer location outside of intron-exon boundary and, when possible, presence in both populations. Information on the first blastp hit against the non-redundant database of each contig was obtained using Blast2GO (Conesa et al., 2005) and used along with blast searches on a list of candidate genes to add SNPs that fell within candidate genes using less restrictive criteria than other SNPs, for example, accepting SNPs unique to one population. Blastclust (http://www. 
csc.fi/english/research/sciences/bioscience/programs/blast/blastclust) was also used to remove contigs aligning to potentially highly repeated sequences, which could cause SNP scoring problems.

\section{Genotyping}

DNA extractions were performed by squashing individual flies in $50 \mu \mathrm{l}$ Squishing buffer (10 mm TrisHCl pH 8.2, 1 mM EDTA, $25 \mathrm{~mm} \mathrm{NaCl}, 200$ $\mathrm{mg} \mathrm{ml}{ }^{-1}$ proteinase $\mathrm{K}$ ), incubating at $50^{\circ} \mathrm{C}$ for $2 \mathrm{~h}$ and boiling for $2 \mathrm{~min}$ to deactivate the proteinase K. DNA clean-up was performed with standard ethanol precipitation (Sambrook and Russell, 2001).

The 384 SNPs were scored on the Illumina BeadXpress platform (Illumina Inc., San Diego, CA, USA) and analysed with GenomeStudio 2010.1 (Genotypic module 1.7.4, Illumina). Individuals were removed if they were associated with genotyping errors (non-Mendelian inheritance in multiple markers), as identified by GenotypeChecker (Paterson and Law, 2011). Genotyping errors cause too many spurious recombination events and inflate genetic maps. Individuals were also removed if they did not have relatives or were missing phenotypic data. After cleaning, 214 and 334 individuals (Vancouver) and 171 and 340 individuals (Oulanka) were included in the QTL analyses for song and $\mathrm{CHC}$, respectively. SNPs were removed from further analysis if they resulted in any parent-offspring mismatches or were obvious outliers in call rate $(<0.80)$. In total, 127 and 130 SNPs were considered of sufficient quality for genetic map construction for Vancouver and Oulanka, respectively, and 79 of them were common between the populations. All data used in the QTL analysis have been submitted to Dryad.

\section{Genetic map construction}

Linkage mapping was performed as in Slate (2008) using CRI-MAP v2.504a (Green et al., 1990; 2009) (http://www.animalgenome.org/tools/share/crimap/). The pedigrees were first split into subfamilies using the CRIGEN command, and linkage groups were initially defined by the AUTOGROUP command. Linked markers were identified using the TWOPOINT command, with all pairs of markers producing logarithm of the odds (LOD) scores in excess of 3.0 being regarded as linked. For each linkage group, the most parsimonious marker order was determined using the BUILD, FLIPS, FLIPS3 and FLIPS5 commands. The linkage groups were assigned to the five $D$. montana chromosomes by blasting the sequence of their markers against the $D$. virilis scaffolds, which have been mapped to chromosomes in FlyBase (Altschul et al., 1990; St Pierre et al., 2014). When the linkage maps had a different marker order between the populations (chromosomes 2 and 4), the difference in log likelihood of the collinear marker order and the parsimonious marker order was obtained for the shared markers using CHROMPIC and was always $>3$ (40.06 and 38.13, respectively), providing support that the markers are not collinear. The genetic maps were plotted using MAPCHART v2.2 (Voorrips, 2002).

Marker 12716_321_id was placed by CRI_MAP $100 \mathrm{~cm}$ away from the next marker at one end of the Oulanka $\mathrm{X}$ chromosome. We retained the marker, by reducing the distance to $49.5 \mathrm{~cm}$, for three reasons: It mapped without problems on the Vancouver $\mathrm{X}$, it has a homologue on the $\mathrm{D}$. virilis $\mathrm{X}$ and there was low marker coverage of the $\mathrm{X}$

\section{Chromosome partitioning analysis}

Genome-wide relatedness matrices (GWRMs), weighted for expected relatedness from pedigree information, were constructed using the methodology of Robinson et al. (2013): For each autosome, pairs of GWRMs were made for its markers and those of the remaining autosomes. Each pair of GWRMs was used, in turn, to partition the variance explained by an autosome. To determine the additive genetic variation explained by an autosome, we compared the likelihood of a model with both GWRMs and a model with only the GWRM of the remaining autosomes for each GWRM pair. A separate GWRM was constructed for the $\mathrm{X}$ chromosome and was only used to estimate the variance explained by the $\mathrm{X}$, in a similar manner. The models included temperature for song and sex for CHC data as fixed effects. Model fitting was performed in ASReml version 3 (Gilmour et al., 2009). Full details of the methodology are available in Santure et al. (2013).

If a trait is polygenic, the variance explained by each chromosome should be proportional to its size. We considered three proxies of gene number per chromosome: the physical length of the $D$. virilis homologous chromosome and the genetic map length of the chromosome in either $D$. montana or D. virilis. We consider the $D$. virilis chromosome physical length to be the best proxy because loci on the $\mathrm{X}$ chromosome were underrepresented in our genetic maps, making them shorter than expected. Possible reasons for the low SNP density on the $\mathrm{X}$ include the fact that males, which provided about half the RNA used in sequencing, are hemizygous (rare SNPs would be less likely to be retained by the SNP-filtering pipeline). The D. virilis chromosome lengths were obtained from Flybase (Altschul et al., 1990; St Pierre et al., 2014), by summing up the lengths of all the $D$. virilis scaffolds, that have been mapped to each chromosome.

\section{QTL—animal model analysis}

Identity by descent matrices were constructed at 10-cm intervals for all individuals of each pedigree, using all SNPs on the relevant chromosome with a Markov chain Monte Carlo approach implemented in LOKI v2.4.5 (Heath et al., 1997; Heath, 1997), and a parameter setting of 10000 iterations for each position. The $\mathrm{X}$ chromosome is hemizygous in males, but LOKI requires two alleles for all markers. We manually made the male X marker information compatible with the analysis by adding a novel allele for each $\mathrm{X}$ male genotype (that is, all males carried one copy of a dummy allele that was absent in females). The phenotypic variance was partitioned into fixed and random effects in a restricted maximum-likelihood (REML) framework using ASReml version 3 (Gilmour et al., 2009), as in Slate (2005). Heritability $\left(\mathrm{h}^{2}\right)$ was estimated as the ratio of additive genetic to total phenotypic variance from a polygenic model. Potential QTL effects were estimated as in Santure et al. (2013). Briefly, a LOD score was calculated by subtracting the log-likelihood of a polygenic model from the log-likelihood of a polygenic plus QTL model, the latter also including the identity by descent matrix, for each genomic interval. Significance thresholds were adjusted for multiple testing based on genome size, following Lander and Kruglyak (1995). Two genome-wide linkage thresholds were calculated for each population (Supplementary File S1): the suggestive linkage threshold is expected once per genome scan, while the significant linkage threshold has a $1 / 20$ false-positive rate, that is, it represents a genomewide significance of $P<0.05$ (Lander and Kruglyak, 1995; Nyholt, 2000).

We could not directly compare the two populations within a single analysis because not all of their markers were shared, and because the relative positions of the markers differed. Instead, we indirectly compared the populations by comparing results from the chromosome partitioning analysis, which is not affected by marker order.

\section{Power analysis}

We performed simulations using custom scripts to estimate the probability of detecting, within the Vancouver pedigree, a QTL of similar magnitude to the one detected in the inter-population study (Lagisz et al., 2012). We used sexaveraged map distances and simulated a trait with heritability of 0.30 , half the variance of which was caused by a single QTL in the middle of chromosome 2 $\left(\mathrm{V}_{\mathrm{a}}=0.15, \mathrm{~V}_{\mathrm{qtl}}=0.15, \mathrm{~V}_{\text {residual }}=0.70\right)$. The simulations were run for 100 replicates, assumed all individuals were phenotyped and used the same suggestive and significant linkage thresholds as the experimental QTL analysis.

\section{RESULTS}

\section{Phenotypic variation}

Phenotypic variation for the two pedigrees has been described before and the populations differ in all traits (Veltsos et al., 2012). Means and standard deviations for all phenotyped traits are presented in Table 1.

\section{Genetic maps}

The total length of the map from the Vancouver pedigree was $789.8 \mathrm{~cm}$, and that from the Oulanka pedigree $689.8 \mathrm{~cm}$. Comparison of these maps clearly shows a different marker order for chromosomes 2 and 4 (Figure 1, Supplementary File S2). Chromosomal rearrangements are known to have occurred within D. montana and we therefore believe that extensive changes in gene order are not unlikely. A population on the Western Coast of North America (around 
Vancouver) has at least one inversion on the second chromosome and 10 inversions on the fourth chromosome that are not found in Finland, while the Finnish population has 3 inversions on the second chromosome that are not found in the Vancouver region (Hsu, 1952; Patterson and Stone, 1952; Moorhead, 1954; Morales-Hojas et al., 2007). Information on the markers such as heterozygosities, their top blast hit, potential functions and sequence of the relevant contig is provided in Supplementary File S3.

\section{Heritability estimates}

Heritability and standard error estimates based on the relatedness matrices calculated from all SNPs are shown in Table 1. The estimates are broadly consistent between the populations, suggesting some similarities between the traits (correlation coefficient between heritability estimates for PCs $=0.83$ ).

\section{Power analysis}

Simulations suggest there was only moderate power to detect QTLs of an effect size similar to those detected in between-population crosses. Out of 100 simulations, 55 detected the simulated QTL at the suggestive threshold and only 13 detected it at the genome-wide significance threshold (Supplementary File S4a). When detected, the

Table 1 Trait values (genotyped individuals from Veltsos et al. 2012) and heritability estimates from polygenic models of song and $\mathrm{CHC}$ traits

\begin{tabular}{lrrrrr}
\hline & \multicolumn{2}{c}{ Value \pm s.d. } & & \multicolumn{2}{c}{$h^{2} \pm$ s.e. } \\
\cline { 2 - 3 } \cline { 5 - 6 } & Oulanka & Vancouver & & Oulanka & Vancouver \\
\hline FRE (Hz) & $253 \pm 35$ & $235 \pm 39$ & & $0.08 \pm 0.11$ & $0.11 \pm 0.09$ \\
IPI (msec) & $40.18 \pm 6.38$ & $43.47 \pm 6.83$ & & $0.2 \pm 0.14$ & $0.21 \pm 0.11$ \\
PN & $4.80 \pm 0.95$ & $4.79 \pm 0.85$ & & $0.14 \pm 0.12$ & $0.06 \pm 0.1$ \\
CN & $9.40 \pm 1.25$ & $10.13 \pm 1.40$ & & $0.52 \pm 0.18$ & $0.15 \pm 0.12$ \\
Song_pc1 & $0.39 \pm 1.16$ & $-0.41 \pm 1.34$ & & $0.14 \pm 0.13$ & $0.11 \pm 0.10$ \\
Song_pc2 & $-0.02 \pm 1.05$ & $0.14 \pm 1.00$ & & $0.28 \pm 0.14$ & $0.17 \pm 0.10$ \\
Song_pc3 & $0.16 \pm 0.93$ & $-0.09 \pm 0.90$ & & $0.4 \pm 0.17$ & $0.25 \pm 0.14$ \\
Song_pc4 & $-0.03 \pm 0.46$ & $0.04 \pm 0.48$ & & NA & $0.16 \pm 0.10$ \\
CHC_MF_pc1 & $1.19 \pm 2.19$ & $-1.34 \pm 2.91$ & & $0.02 \pm 0.06$ & $0.05 \pm 0.05$ \\
CHC_MF_pc2 & $-0.90 \pm 0.93$ & $1.14 \pm 1.47$ & & $0.56 \pm 0.08$ & $0.37 \pm 0.09$ \\
CHC_MF_pc3 & $-0.19 \pm 1.08$ & $0.19 \pm 1.42$ & & $0.35 \pm 0.09$ & $0.06 \pm 0.05$ \\
CHC_MF_pc4 & $0.38 \pm 0.78$ & $-0.45 \pm 1.04$ & & $0.12 \pm 0.07$ & $0.1 \pm 0.06$ \\
\hline
\end{tabular}

Abbreviations: $\mathrm{CHC}$, cuticular hydrocarbon; $\mathrm{CN}$, cycle number per pulse; FRE, frequency; IPI, inter-pulse interval; NA, not available; PN, pulse number.
Beavis Effect (Göring et al., 2001) was observed, that is, the QTL effect size was usually overestimated (mean effect size was 0.25 instead of simulated 0.15 proportion of total variance). When the QTL location was detected, it was accurately mapped (Supplementary File S4b).

\section{QTL results}

On the basis of the total genetic map length, the suggestive and significant LOD scores for Vancouver were 1.12 and 2.62, respectively, and for Oulanka 1.05 and 2.56, respectively. We did not detect genome-wide significant results in the QTL analysis but observed suggestive linkage once, on chromosome 5, for $\mathrm{CN}$ in Oulanka (Figure 2). The result had a slightly higher LOD score when song PCs were used, (song_pc3 which is strongly influenced by CN (Veltsos et al., 2012)), but was otherwise qualitatively similar (Supplementary File S6). Interestingly, the song characters mostly influencing song_pc3 (FRE, IPI and CN (Veltsos et al., 2012)) had lower LRT (log likelihood ratio test) values individually than the LRT for the PC, suggesting that multivariate analysis may increase detection power, presumably because a PC axis reduces the noise of individual measurements.

\section{Genetic variation partitioning}

There was no relationship between percent variance explained and chromosome length, regardless of the proxy of chromosome length used (Table 2, Supplementary File S5). It is difficult to detect such a correlation because of the small number of data points as there are only five chromosomes in D. montana. In addition, it is difficult to detect differences in percent variance explained between the chromosomes because they have similar sizes, which may make the between-chromosome variance small, relative to the withinchromosome error in estimating variance. There were 18 cases where a single chromosome explained non-zero variance (Table 3). No formal test of significance was performed because the null hypothesis of no variation explained by any chromosome is false for polygenic heritable traits.

\section{Population comparison}

We compared the genetic architecture between the populations by plotting the percent variance explained by each chromosome for each trait, in the two populations (Supplementary File S7). Although the values were often similar, there were occasions where a chromosome clearly explained more variance in one population than in the other population (for example, for song_pc1).

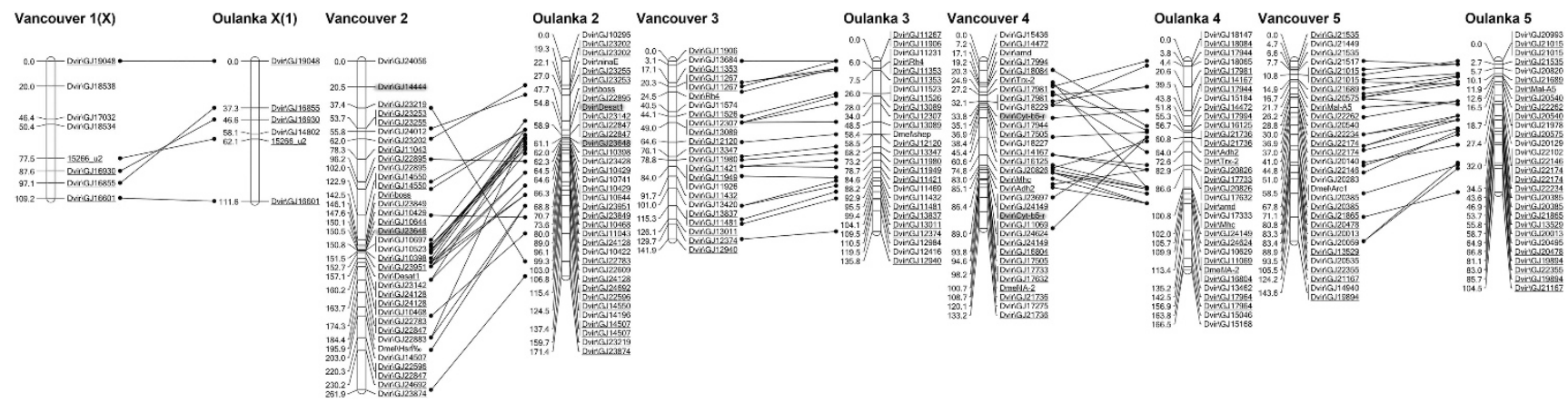

Figure 1 Comparison of genetic maps from the two populations. The markers are represented by the name of the top $D$. virilis blast hit of the relevant contig. Chromosome 2 and 4 are clearly not collinear between the two populations. Candidate genes are indicated by shading: Dvir/GJ14444-Ebony, Dvir/GJ23648Slowpoke, Dvir/Desat1-DsatF and Dvir/GJCyt-b5-r-CytB5. 
Oulanka Song QTL

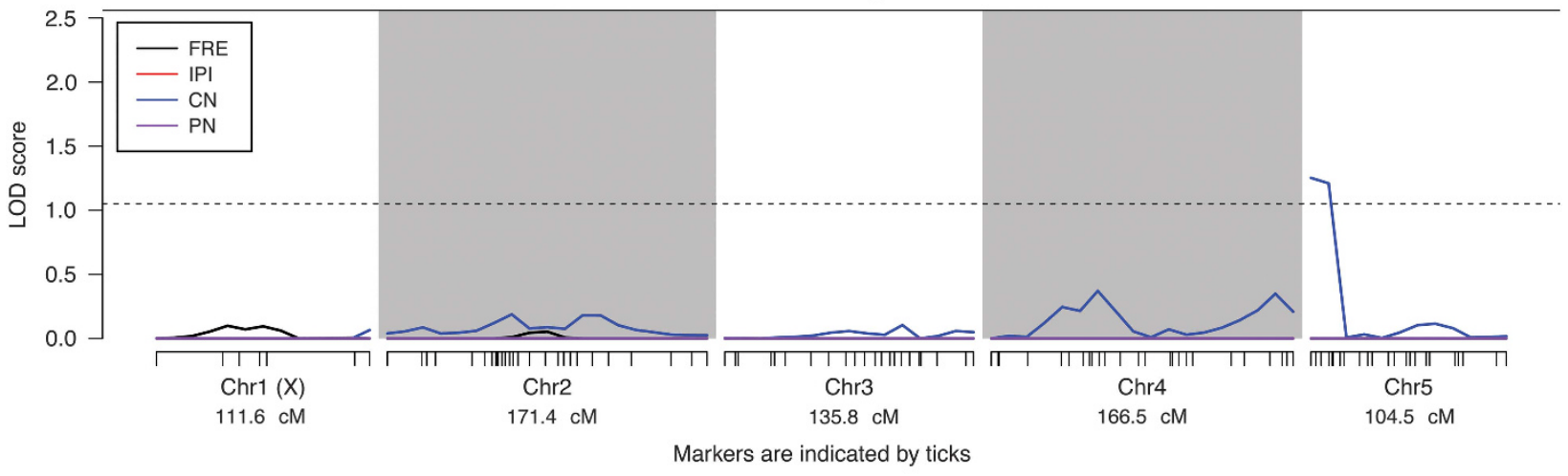

Vancouver Song QTL

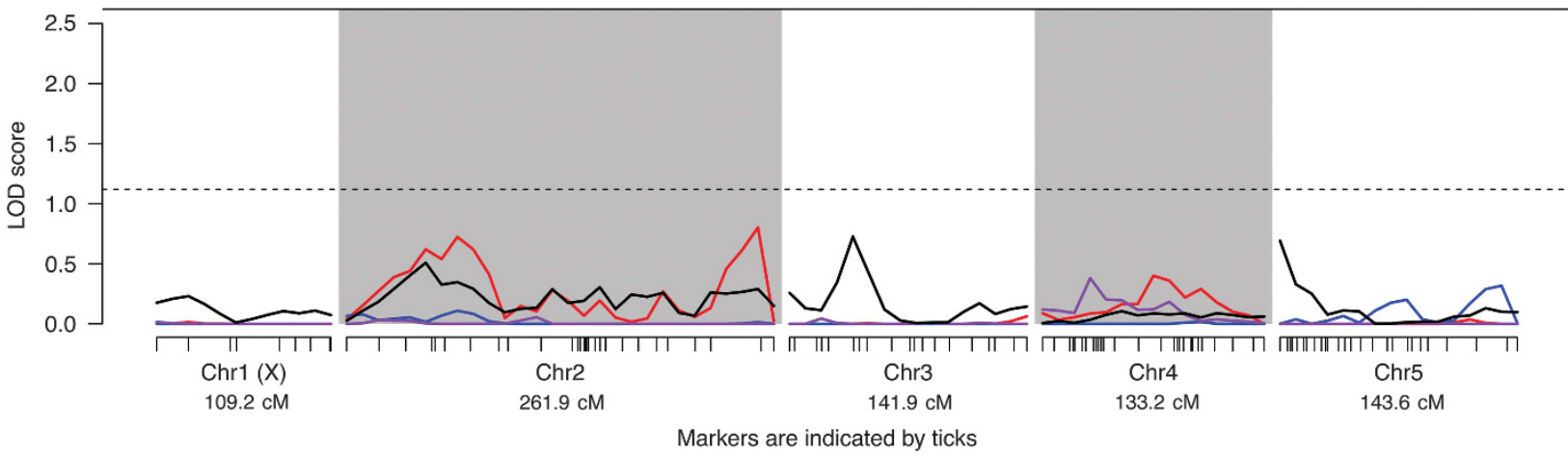

Oulanka CHC PC QTL

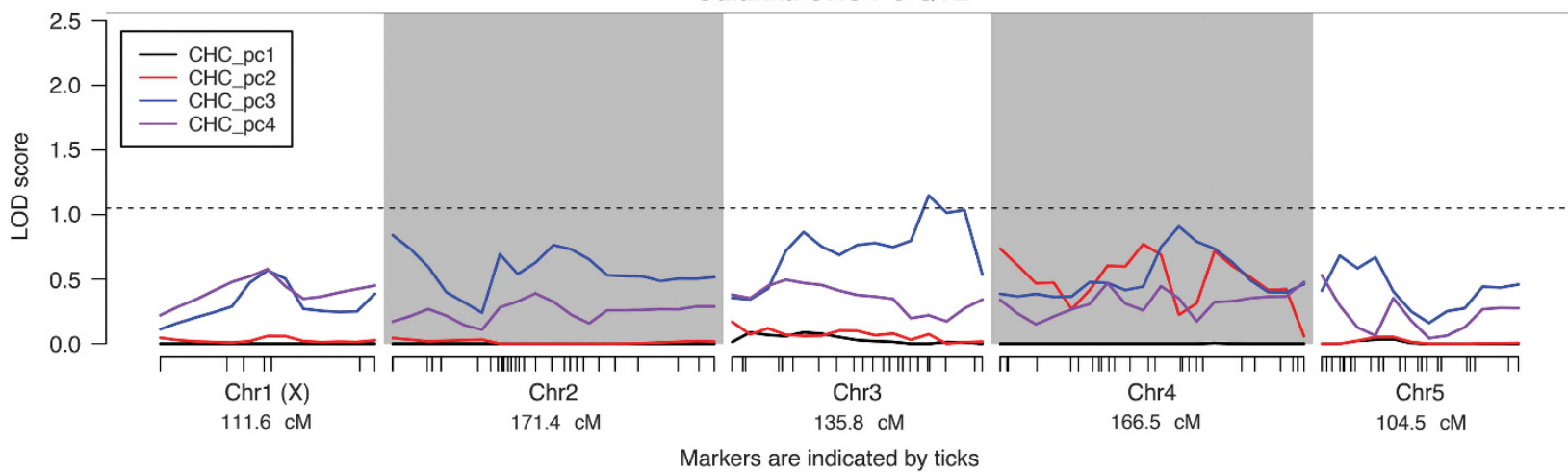

Vancouver CHC PC QTL

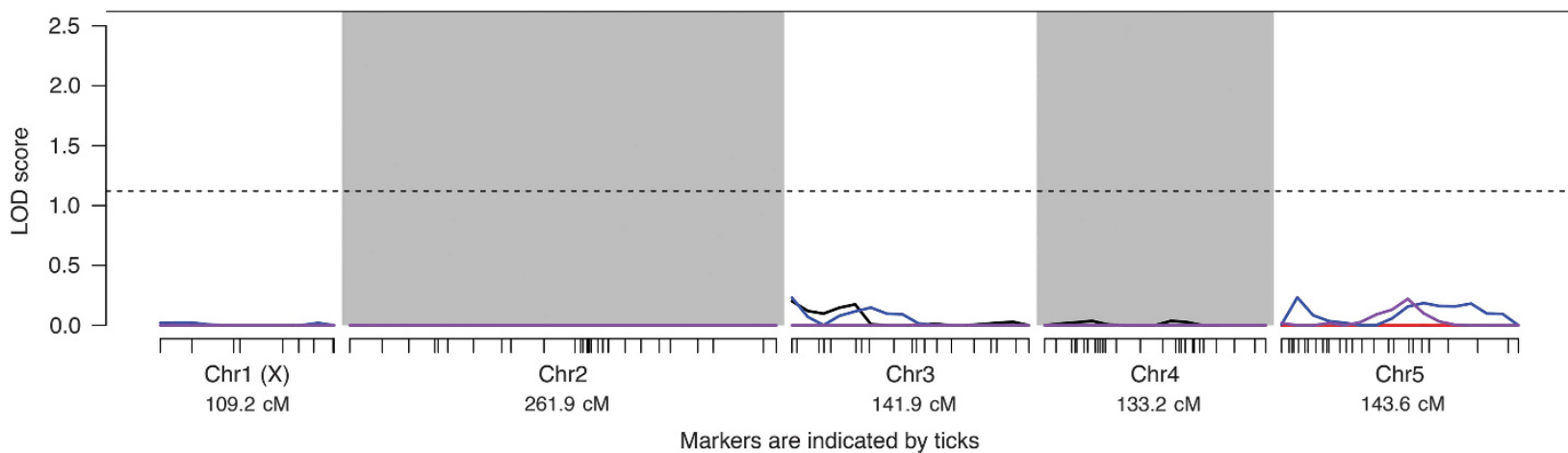

Figure 2 QTL maps of song and CHCs in the two populations. The dotted and uninterrupted horizontal lines indicate suggestive and significant linkage, respectively. Chromosomes X and 2, which showed QTL for song in the between-population study, have no QTL identified. 
Table 2 Correlation between PVE and D. virilis chromosome length

\begin{tabular}{lcc}
\hline Character & Correlation & $\mathrm{P}$ \\
\hline FRE & -0.52 & $\mathrm{NS}$ \\
IPI & 0.47 & 0.17 \\
PN & -0.04 & $\mathrm{NS}$ \\
CN & -0.50 & $\mathrm{NS}$ \\
song_pc1 & 0.51 & 0.13 \\
song_pc2 & -0.30 & $\mathrm{NS}$ \\
song_pc3 & -0.41 & $\mathrm{NS}$ \\
song_pc4 & -0.53 & $\mathrm{NS}$ \\
CHC_pc1 & -0.19 & 0.60 \\
CHC_pc2 & 0.28 & 0.43 \\
CHC_pc3 & -0.52 & $\mathrm{NS}$ \\
CHC_pc4 & -0.3 & $\mathrm{NS}$ \\
\hline
\end{tabular}

Abbreviations: $\mathrm{CHC}$, cuticular hydrocarbon; CN, cycle number per pulse; FRE, frequency; IPI, inter-pulse interval; PN, pulse number; PVE, percent variance explained. Each chromosome is represented by the combined PVE values from the two pedigrees, because they did not differ significantly (data not shown). $P$-values of negative correlations are labelled as 'NS' because only positive correlations are expected.

\section{DISCUSSION}

We have attempted to identify the architecture of genetic variation available for natural and sexual selection to act upon, within natural populations, by performing heritability and QTL analysis on song and CHC phenotypes using pedigrees established from two populations of D. montana. Although we found significant heritability, we did not find evidence of large effect QTL for any trait. Our results are broadly suggestive of polygenic determination for intrapopulation phenotypic variation, though some individual chromosomes explained more phenotypic variation than others, in a population-specific manner.

Song and CHCs contribute to mating success in D. montana, and CHCs are probably also involved in ecological adaptation (Veltsos et al., 2012; Jennings et al., 2014a). Heritabilities were sometimes low (Table 1), which may well have militated against our ability to detect QTL (the only suggestive QTL found was for a song trait with one of the highest heritabilities, $\mathrm{CN}$ in Oulanka). Nevertheless, our heritability estimates for song were usually larger than previous estimates in D. montana, though sometimes they were not (Aspi and Hoikkala, 1993; Suvanto et al., 1999). The estimates reported here are probably more robust because of the larger sample size, the estimation of relatedness from pedigrees and the reduced measurement noise achieved by using different song analysis methods. The earlier estimation of heritabilities from overwintered flies and the finding that they increase compared with non-overwintered flies (Suvanto et al., 1999) remains important because sexual selection on courtship song often acts only after the flies overwinter as adults.

Simulations suggested that our power to detect QTL was only moderate. The simulated trait effect size was based on a QTL detected for song variation in crosses between North American and Finnish $D$. montana (Colorado and Oulanka, that is, a different population from North America) (Mirol et al., 2007; Schafer et al., 2010; Lagisz et al., 2012). We failed to detect QTL for the same traits and genomic positions as the between-population study. These were for IPI and pulse number on the $\mathrm{X}$ chromosome, and FRE, CN and IPI on chromosome 2 (Lagisz et al., 2012). We then attempted to implicate the same chromosomes using the chromosome-partitioning analysis. Of the above traits, a large proportion of variance $(31.8 \pm 17.3 \%)$ was only found to be predicted for IPI on chromosome 2 in Oulanka (Supplementary File S7). One possible explanation for the disparity between the studies is that large-effect alleles that differ between clades are not making a major contribution to genetic variation within
Table 3 Traits where a chromosome explains non-zero variance (percent varience explained-s.e. $>0$ )

\begin{tabular}{|c|c|c|c|}
\hline Chromosome & Character & PVE \pm s.e. & Population \\
\hline \multirow[t]{2}{*}{2} & song_pc1 & $0.30 \pm 0.17$ & 0 \\
\hline & & $0.00 \pm 0.00$ & v \\
\hline \multirow[t]{2}{*}{2} & IPI & $0.32 \pm 0.17$ & 0 \\
\hline & & $0.00 \pm 0.00$ & V \\
\hline \multirow[t]{2}{*}{2} & CHC_pc2 & $0.10 \pm 0.09$ & 0 \\
\hline & & $0.07 \pm 0.08$ & v \\
\hline \multirow[t]{2}{*}{3} & song_pc3 & $0.35 \pm 0.16$ & 0 \\
\hline & & $0.04 \pm 0.07$ & v \\
\hline \multirow[t]{2}{*}{3} & PN & $0.10 \pm 0.12$ & 0 \\
\hline & & $0.16 \pm 0.12$ & V \\
\hline \multirow[t]{2}{*}{3} & FRE & $0.00 \pm 0.00$ & 0 \\
\hline & & $0.13 \pm 0.11$ & v \\
\hline \multirow[t]{2}{*}{3} & $\mathrm{CN}$ & $0.19 \pm 0.17$ & 0 \\
\hline & & $0.03 \pm 0.07$ & v \\
\hline \multirow[t]{2}{*}{3} & CHC_pc3 & $0.03 \pm 0.07$ & 0 \\
\hline & & $0.09 \pm 0.07$ & v \\
\hline \multirow[t]{2}{*}{4} & $\mathrm{CN}$ & $0.22 \pm 0.16$ & 0 \\
\hline & & $0.04 \pm 0.07$ & V \\
\hline \multirow[t]{2}{*}{4} & CHC_pc3 & $0.00 \pm 0.00$ & 0 \\
\hline & & $0.03 \pm 0.05$ & V \\
\hline \multirow[t]{2}{*}{4} & CHC_pc1 & $0.00 \pm 0.00$ & 0 \\
\hline & & $0.08 \pm 0.07$ & V \\
\hline \multirow[t]{2}{*}{4} & IPI & $0.15 \pm 0.13$ & 0 \\
\hline & & $0.07 \pm 0.08$ & V \\
\hline \multirow[t]{2}{*}{4} & song_pc2 & $0.15 \pm 0.14$ & 0 \\
\hline & & $0.00 \pm 0.00$ & V \\
\hline \multirow[t]{2}{*}{5} & song_pc2 & $0.00 \pm 0.00$ & 0 \\
\hline & & $0.29 \pm 0.12$ & V \\
\hline \multirow[t]{2}{*}{5} & CHC_pc3 & $0.07 \pm 0.07$ & 0 \\
\hline & & $0.00 \pm 0.00$ & V \\
\hline \multirow[t]{2}{*}{5} & CHC_pc2 & $0.07 \pm 0.07$ & 0 \\
\hline & & $0.17 \pm 0.08$ & v \\
\hline \multirow[t]{2}{*}{5} & IPI & $0.04 \pm 0.13$ & 0 \\
\hline & & $0.10 \pm 0.09$ & v \\
\hline \multirow[t]{2}{*}{5} & song_pc1 & $0.07 \pm 0.12$ & 0 \\
\hline & & $0.11 \pm 0.10$ & v \\
\hline
\end{tabular}

Abbreviations: $\mathrm{CHC}$, cuticular hydrocarbon; $\mathrm{CN}$, cycle number per pulse; FRE, frequency; IPI, inter-pulse interval; PN, pulse number; PVE, percent variance explained. In all cases, only one of the populations explains > O PVE.

populations. This supports recent studies suggesting that the genetic architecture of intra-population and inter-clade variation may differ for Drosophila song (Gleason, 2005; Arbuthnott, 2009) and for sexually selected traits generally (Chenoweth and McGuigan, 2010). Similarly, some between-population QTL may be due to Coloradospecific alleles of large effect, which were not sampled in this study. An alternative explanation for the disparity between the within- and between-population studies may be the greater range in phenotypes amongst the crosses between two populations, compared with withinpopulation variation. Colorado flies are different from those from Vancouver, in both their songs and pheromones (Klappert et al., 2007; Routtu et al., 2007; Jennings et al., 2014a), despite coming from the same phylogeographic clade.

A third possibility is that QTLs of large effect are difficult to detect using intra-population analysis. When detected in crosses between lines, QTL may be caused by variants of large effect that are at low frequency within the population. Variants of large effect under selection would rapidly fix within a population during adaptation to 
new environments and are not likely to segregate within populations (Scoville et al., 2011). Consequently, they may be readily associated with large QTL in between-population crosses that do not explain much phenotypic variance at the population level.

A fourth possibility is that the between-population QTL seem large because fixed chromosomal rearrangements between the populations caused a reduction in recombination in some genomic regions, effectively combining the effects of many loci of small effect, and causing them to segregate as a single locus. Loci implicated in repeated evolution of adaptive traits are often associated with inversions (Martin and Orgogozo, 2013). When the genes captured by an inversion are involved in reproductive isolation, the inversion may become important in maintaining isolation in the face of gene flow (Noor et al., 2001; Kirkpatrick and Barton, 2006). The IPI QTL in an inversion on chromosome 2 in the between-population study (Lagisz et al., 2012) is compatible with both ideas of capturing multiple genes and being associated with reproductive isolation, because IPI is under sexual selection and differs between species (Saarikettu et al., 2005b; Veltsos et al., 2012). Within-population crosses would not be affected by such inversions, which could explain the failure to detect QTL.

The QTL in sexually selected traits in within- and betweenpopulation studies are usually different (Arbuthnott, 2009; Chenoweth and McGuigan, 2010). One exception, for a pre-mating signal, involves a pheromone QTL in the moth species Heliothis subflexa and $H$. virescens (Groot et al., 2013). Few studies have compared the magnitude of effect sizes within and between populations in addition to assessing whether the same QTLs are implicated, and it may be that consistent genetic effects are associated with phenotypes where large effect loci are more likely to be found, such as pheromone polymorphisms or genes influencing melanism (Wittkopp et al., 2009).

\section{Comparison between populations}

One common method to demonstrate polygenic variation is through a correlation of percent variance explained with chromosome size. The relatively low number of similarly sized chromosomes makes this an ineffective test for Drosophila, but most chromosomes explained some variation, which is compatible with polygenic genetic architecture. There were cases where one chromosome explained more variation than others. This sometimes happened in a population-specific manner (Supplementary File S7), providing evidence of a subtly different genetic architecture between the Oulanka and Vancouver populations.

The X chromosome had the lowest number of markers in our study even though it is the largest chromosome. The low X coverage would be particularly unfortunate if there is a large $\mathrm{X}$ effect, that is if the $\mathrm{X}$ is disproportionately influencing reproductive isolation (Presgraves, 2008) or genes under sexual selection (Qvarnstrom and Bailey, 2009; Dean and Mank, 2014), which may have further hindered our ability to detect segregating genetic variation for these traits.

\section{CONCLUSION}

In performing this study, we have developed useful resources, including a comparative transcriptomic data set and a set of SNP markers and associated genetic maps for D. montana. Like many studies of wild populations, our study suffers from limited statistical power. Investigating the genetic architecture of within-population variation probably requires sample sizes in the order of a few thousand, to ensure sufficient statistical power (Rockman, 2012; Slate, 2013). Despite significant heritabilities of the traits, we found limited evidence of common genetic architecture in within-population phenotypic variation and interspecific differences, extending the trend found in other comparisons (Arbuthnott, 2009; Chenoweth and McGuigan, 2010). This may suggest that large effect alleles fixed between populations are not major contributors to variation within populations. It is important to study within-population variation despite such difficulties, because it is the variation upon which natural and sexual selection act, making it the most relevant variation for immediate responses to selection (Prokop et al., 2012). Concentrating on QTL mapping populations from between-species crosses can reveal genetic mechanisms of species differences, but such genes may have diverged in frequency or become fixed after speciation. Understanding sources of genetic variation across the speciation continuum remains a major challenge of evolutionary genetics (The Marie Curie SPECIATION Network, 2012).

\section{DATA ARCHIVING}

Phenotype, genotype and pedigree data as well as raw reads and the assembled transcriptome are available from the Dryad Digital Repository doi:10.5061/dryad.4p9j3.

\section{CONFLICT OF INTEREST}

The authors declare no conflict of interest.

\section{ACKNOWLEDGEMENTS}

The work was supported by the National Environment Research Council (grant NE/E015255/1 to MGR and RKB) and the Academy of Finland (project 132619 to AH). We would like to thank Juan Galindo and Anna Santure for bioinformatic and statistical help. We are grateful to Dario Beraldi for providing a script to streamline the pedigree analysis and parse files between PedigreeChecker, CRIMAP and ASREML, and Matthew Robinson for providing scripts allowing the chromosome partitioning analysis.

Altschul SF, Gish W, Miller W, Myers EW, Lipman DJ (1990). Basic local alignment search tool. J Mol Biol 215: 403-410.

Arbuthnott D (2009). The genetic architecture of insect courtship behavior and premating isolation. Heredity 103: 15-22.

Aspi J (1992). Incidence and adaptive significant of multiple mating in females of two boreal Drosophila virilis-group species. Annales Zoologici Fennici 29: 147-159.

Aspi J, Hoikkala A (1993). Laboratory and natural heritabilities of male courtship song characters in Drosophila montana and D. littoralis. Heredity 70: 400-406.

Aspi J, Hoikkala A (1995). Male mating success and survival in the field with respect to size and courtship song characters in Drosophila littoralis and D. montana (Diptera, Drosophilidae). Journal of Insect Behavior 8: 67-87.

Chenoweth SF, McGuigan K (2010). The genetic basis of sexually selected variation. Annu Rev Ecol Evol Syst 41: 81-101.

Conesa A, Gotz S, Garcia-Gomez JM, Terol J, Talon M, Robles M (2005). Blast2GO: a universal tool for annotation, visualization and analysis in functional genomics research. Bioinformatics 21: 3674-3676.

Davies G, Tenesa A, Payton A, Yang J, Harris SE, Liewald D et al. (2011). Genome-wide association studies establish that human intelligence is highly heritable and polygenic. Mol Psychiatry 16: 996-1005.

Dean R, Mank JE (2014). The role of sex chromosomes in sexual dimorphism: discordance between molecular and phenotypic data. J Evol Biol 27: 1443-1453.

Fitzpatrick MJ, Ben-Shahar Y, Smid HM, Vet LEM, Robinson GE, Sokolowski MB (2005). Candidate genes for behavioural ecology. Trends Ecol Evol (Amst) 20: 96-104.

Gilmour AR, Gogel MJ, Cullis BR, Thompson R (2009). ASReml User Guide Release 3.0. Hemel Hempstead, UK: VSN International Ltd.

Gleason JM (2005). Mutations and natural genetic variation in the courthsip song of Drosophila. Behav Genet 35: 265-277.

Göring HHH, Terwilliger JD, Blangero J (2001). Large upward bias in estimation of locusspecific effects from genomewide scans. Am J Hum Genet 69: 1357-1369.

Green P, Falls K, Crooks S (1990). CRI-MAP 2.4 documentation.

Green P, Falls K, Crooks S (2009). CRI-MAP 2.503 documentation.

Groot AT, Staudacher H, Barthel A, Inglis O, Schofl G, Santangelo RG et al. (2013). One quantitative trait locus for intra- and interspecific variation in a sex pheromone. $\mathrm{Mol} \mathrm{Ecol}$ 22: $1065-1080$.

Heath SC (1997). Markov chain Monte Carlo segregation and linkage analysis for oligogenic models. Am J Hum Genet 61: 748-760.

Heath SC, Snow GL, Thompson EA (1997). MCMC segregation and linkage analysis. Genet Epidemiol 14: 1011-1016. 
Hoikkala A, Aspi J, Suvanto L (1998). Male courtship song frequency as an indicator of male genetic quality in an insect species, Drosophila montana. Proc Biol Sci 265 503-508.

Hoikkala A, Päällysaho S, Aspi J, Lumme J (2000). Localization of genes affecting species differences in male courtship song between Drosophila virilis and D. littoralis. Genet Res 75: $37-45$.

Hsu TC (1952). Chromosomal variation and evolution in the virilis group of Drosophila. University of Texas Publication 52004: 35-72.

Jennings J, Etges WJ, Schmitt T, Hoikkala A (2014a). Cuticular hydrocarbons of Drosophila montana: Geographic variation, sexual dimorphism and potential roles as pheromones. J Insect Physio/ 61: 16-24.

Jennings J, Mazzi D, Ritchie M, Hoikkala A (2011). Sexual and postmating reproductive isolation between allopatric Drosophila montana populations suggest speciation potential. BMC Evol Biol 11: 68.

Jennings J, Snook RR, Hoikkala A (2014b). Reproductive isolation among allopatric Drosophila montana populations. Evolution 68: 3095-3108.

Johnston SE, McEwan JC, Pickering NK, Kijas JW, Beraldi D, Pilkington JG et al. (2011). Genome-wide association mapping identifies the genetic basis of discrete and quantitative variation in sexual weaponry in a wild sheep population. Mol Ecol 20 : 2555-2566.

Kirkpatrick M, Barton NH (2006). Chromosome inversions, local adaptation and speciation. Genetics 173: 419-434.

Klappert K, Mazzi D, Hoikkala A, Ritchie M (2007). Male courtship song and female preference variation between phylogeographically distinct populations of Drosophila montana. Evolution 61: 1481-1488.

Kruuk LE, Hill WG (2008). Introduction. Evolutionary dynamics of wild populations: the use of long-term pedigree data. Proc Biol Sci 275: 593-596.

Lagisz M, Wen S-Y, Routtu J, Klappert K, Mazzi D, Morales-Hojas R et al. (2012). Two distinct genomic regions, harbouring the period and fruitless genes, affect male courtship song in Drosophila montana. Heredity 108: 602-608.

Lander E, Kruglyak L (1995). Genetic dissection of complex traits: guidelines for interpreting and reporting linkage results. Nat Genet 11: 241-247.

Liimatainen JO, Hoikkala A (1998). Interactions of the males and females of three sympatric Drosophila virilis-group species, D. montana, D. littoralis, and D. lummei, (Diptera: Drosophilidae) in intra- and interspecific courtships in the wild and in the laboratory. Journal of Insect Behavior 11: 399-417.

Liimatainen J, Hoikkala A, Aspi J, Welbergen P (1992). Courtship in Drosophila montana: the effects of male auditory signals on the behaviour of flies. Animal Behaviour 43 $35-48$.

Martin A, Orgogozo V (2013). The Loci of repeated evolution: a catalog of genetic hotspots of phenotypic variation. Evolution 67: 1235-1250.

Mirol PM, Schafer MA, Orsini L, Routtu J, Schlötterer C, Hoikkala A et al. (2007). Phylogeographic patterns in Drosophila montana. Mol Ecol 16: 1085-1097.

Moorhead PS (1954). Chromosome variation in giant forms of Drosophila montana University of Texas Publication 5422: 106-129.

Morales-Hojas R, Päällysaho S, Vieira C, Hoikkala A, Vieira J (2007). Comparative polytene chromosome maps of D. montana and D. virilis. Chromosoma 116: 21-27.

Noor MA, Grams KL, Bertucci LA, Reiland J (2001). Chromosomal inversions and the reproductive isolation of species. Proc Natl Acad Sci USA 98: 12084-12088.

Nyholt D (2000). All LODs are not created equal. Am J Hum Genet 67: 282-288.

Parker DJ, Vesala L, Ritchie M, Laiho A, Hoikkala A, Kankare M (2015). How consistent are the transcriptome changes associated with cold acclimation in two species of the Drosophila virilis group? Heredity 115: 13-21.

Paterson T, Law A (2011). Genotypechecker: an interactive tool for checking the inheritance consistency of genotyped pedigrees. Anim Genet 42: 560-562.

Patterson JT, Stone WS (1952). Evolution in the Genus Drosophiila. MacMillan: New York

Päällysaho S, Aspi J, Liimatainen J, Hoikkala A (2003). Role of X chromosomal song genes in the evolution of species-specific courtship songs in Drosophila virilis group species. Behav Genet 33: 25-32.

Presgraves DC (2008). Sex chromosomes and speciation in Drosophila. Trends Genet 24: 336-343.

Prokop ZM, Michalczyk $Ł$, Drobniak SM, Herdegen M, Radwan J (2012). Meta-analysis suggests choosy females get sexy sons more than 'good genes'. Evolution 66: 2665-2673

Qvarnstrom A, Bailey R (2009). Speciation through evolution of sex-linked genes. Heredity 102: 4-15.

Ritchie M, Etges WJ, de Oliveira CC, Gragg E, Ortiz-Barrientos D, Noor M (2007). Genetics of incipient speciation in Drosophila mojavensis. I. Male courtship song, mating success, and genotype x environment interactions. Evolution 61: 1481-1488.
Ritchie M, Saarikettu M, Livingstone S, Hoikkala A (2001). Characterization of female preference functions for Drosophila montana courtship song and a test of the temperature coupling hypothesis. Evolution 55: 721-727.

Robinson MR, Santure AW, DeCauwer I, Sheldon BC, Slate J (2013). Partitioning of genetic variation across the genome using multimarker methods in a wild bird population. $\mathrm{Mol}$ Ecol 22: 3963-3980.

Rockman MV (2012). The QTN program and the alleles that matter for evolution: All that's gold does not glitter. Evolution 66: 1-17.

Routtu J, Mazzi D, der Linde Van K, Mirol P, Butlin RK, Hoikkala A (2007). The extent of variation in male song, wing and genital characters among allopatric Drosophila montana populations. J Evol Biol 20: 1591-1601

Rundle HD, Blows MW, Chenoweth SF (2009). The diversification of mate preferences by natural and sexual selection. J Evol Biol 22: 1608-1615.

Saarikettu M, Liimatainen JO, Hoikkala A (2005a). The role of male courtship song in species recognition in Drosophila montana. Behav Genet 35: 257-263.

Saarikettu M, Liimatainen JO, Hoikkala A (2005b). Intraspecific variation in mating behaviour does not cause sexual isolation between Drosophila virilis strains. Animal Behaviour 70: 417-426.

Safran RJ, Scordato ESC, Symes LB, Rodríguez RL, Mendelson TC (2013). Contributions of natural and sexual selection to the evolution of premating reproductive isolation: research agenda. Trends Ecol Evol (Amst) 28: 643-650.

Salminen T, Vesala L, Hoikkala A (2012). Photoperiodic regulation of life-history traits before and after eclosion: egg-to-adult development time, juvenile body mass and reproductive diapause in Drosophila montana. J Insect Physiol 58: 1541-1547.

Sambrook J, Russell D (2001). Molecular Cloning: A Laboratory Manual. Cold Spring Harbor Laboratory Press.

Santure AW, De Cauwer I, Robinson MR, Poissant J, Sheldon BC, Slate J (2013). Genomic dissection of variation in clutch size and egg mass in a wild great tit (Parus major) population. Mol Ecol 22: 3949-3962.

Schafer M, Mazzi D, Klappert K, Kauranen H, Vieira J, Hoikkala A et al. (2010). A microsatellite linkage map for Drosophila montana shows large variation in recombination rates, and a courtship song trait maps to an area of low recombination. J Evol Biol 23: $518-527$

Scoville AG, Lee YW, Willis JH, Kelly JK (2011). Explaining the heritability of an ecologically significant trait in terms of individual quantitative trait loci. Biology Letters 7: 896-898.

Slate J (2005). Quantitative trait locus mapping in natural populations: progress, caveats and future directions. $\mathrm{Mol} \mathrm{ECO} / \mathrm{14}$ : 363-379.

Slate J (2008). Robustness of linkage maps in natural populations: a simulation study. Proc Biol Sci 275: 695-702.

Slate J (2013). From beavis to beak colour: A simulation study to examine how much QTL mapping can reveal about the genetic architecture of quantitative traits. $\mathrm{Mol} E \mathrm{col} 67$ $1251-1262$

St Pierre SE, Ponting L, Stefancsik R, McQuilton P FlyBase Consortium (2014). FlyBase 102-advanced approaches to interrogating FlyBase. Nucleic Acids Res 42 D780-D788.

Stern D, Orgogozo V (2008). The loci of evolution: How predictable is genetic evolution? Evolution 62: 2155-2177.

Stern D, Orgogozo V (2009). Is genetic evolution predictable? Science 323: 746-751.

Suvanto L, Liimatainen JO, Hoikkala A (1999). Variability and evolvability of male song characters in Drosophila montana populations. Hereditas 130: 13-18.

The Marie Curie SPECIATION Network (2012). What do we need to know about speciation? Trends Ecol Evol (Amst) 27: 27-39.

Throckmorton LH (1982)15. The virilis species groupInAshburner M, Carson HL, Thompson JN(eds) The Genetics and Biology of Drosophila. Academic Press: London: Londonpp 227-289.

Travisano M, Shaw RG (2013). Lost in the map. Evolution 67: 305-314.

Veltsos P, Wicker-Thomas C, Butlin RK, Hoikkala A, Ritchie M (2012). Sexual selection on song and cuticular hydrocarbons in two distinct populations of Drosophila montana. Ecol Evol 2: 80-94.

Vesala L, Hoikkala A (2011). Effects of photoperiodically induced reproductive diapause and cold hardening on the cold tolerance of Drosophila montana. J Insect Physiol 57: $46-51$

Voorrips RE (2002). MapChart: software for the graphical presentation of linkage maps and QTLs. J Hered 93: 77-78.

Wittkopp PJ, Stewart EE, Arnold LL, Neidert AH, Haerum BK, Thompson EM et al. (2009). Intraspecific polymorphism to interspecific divergence: Genetics of pigmentation in Drosophila. Science 326: 540-544.

Supplementary Information accompanies this paper on Heredity website (http://www.nature.com/hdy) 